\title{
Energy-efficient Computing for HPC Workloads on Heterogeneous Manycore Chips
}

\author{
Akhil Langer, Ehsan Totoni, Udatta S. Palekar ${ }^{\ddagger}$, Laxmikant V. Kalé \\ Department of Computer Science, ${ }^{\ddagger}$ Department of Buisiness Administration \\ University of Illinois at Urbana-Champaign, Illinois, USA \\ \{alanger, totoni2, palekar, kale\}@illinois.edu
}

\begin{abstract}
Power and energy efficiency is one of the major challenges to achieve exascale computing in the next several years. While chips operating at low voltages have been studied to be highly energy-efficient, low voltage operations lead to heterogeneity across cores within the microprocessor chip. In this work, we study chips with low voltage operation and discuss programming systems, and performance modeling in the presence of heterogeneity. We propose an integer linear programming based approach for selecting optimal configuration of a chip that minimizes its energy consumption. We obtain an average of $26 \%$ and $10.7 \%$ savings in energy consumption of the chip for two HPC mini-applications - miniMD and Jacobi, respectively. We also evaluate the energy savings with execution time constraints, using the proposed approach. These energy savings are significantly more than the savings by sub-optimal configurations obtained from heuristics.
\end{abstract}

Categories and Subject Descriptors D.1.3 [Concurrent Programming]: Parallel programming; G.1.6 [Optimization]: Integer programming, Quadratic programming models; F.1.2 [Models of Computation]: Parallelism and concurrency; H.3.4 [Systems and Software]: Performance evaluation (efficiency and effectiveness); C.1.2 [Multiple Data Stream Architectues]: Parallel processors

General Terms Measurement, Performance, Experimentation

Keywords Energy, Power, Optimization, Multicore chips, Low Voltage Computing, Near Threshold Voltage Comput-

Permission to make digital or hard copies of all or part of this work for personal or classroom use is granted without fee provided that copies are not made or distributed for profit or commercial advantage and that copies bear this notice and the full citation on the first page. Copyrights for components of this work owned by others than ACM must be honored. Abstracting with credit is permitted. To copy otherwise, or republish, to post on servers or to redistribute to lists, requires prior specific permission and/or a fee. Request permissions from permissions@acm.org.

PMAM'15, February 7-8, 2015, San Francisco Bay Area, USA

Copyright (c) 2015 ACM 978-1-4503-3404-4/15/02 . . \$15.00.

http://dx.doi.org/10.1145/2712386.2712396 ing, Process Variation, Heterogeneity, Integer Programming, Quadratic Integer Programming

\section{Introduction}

Future microprocessor chips are expected to have variations across the many cores because of the variation in the CMOS manufacturing process. The variation across the chip is expected to further increase with low voltage operation. Chips with low voltage operation have high energy efficiency that is required to build an exascale machine with a power budget of 20MW set by the U.S. Department of Energy. Therefore, it is accepted in the High Performance Computing (HPC) community that there will be heterogeneity across cores of the future generation chips [3]. Frequency and static power consumption of the cores on the same chip can be very different. Low voltage operation can cause up to $50 \%$ variation in frequency across the cores of the same chip. Variation across multiple cores on the same chip can also be obtained on the recent Intel ${ }^{\circledR}$ Haswell chip which allows independent core-frequency scaling, that is, various cores on the same chip can be controlled by the user to run at different frequencies. However, unlike the Intel ${ }^{\circledR}$ Haswell chip, heterogeneity across the cores in a chip with low voltage operation will be forced on to the user (unless all the cores are made to run at a minimum frequency, which will be a very inefficient design).

Energy is one of the biggest challenges faced by the HPC community. Data centers worldwide consumed energy equivalent to 235 billion $\mathrm{KWh}$ in 2010 , which is $2 \%$ of total US electricity consumption. CPU accounts for about $65 \%$ of the total power consumption of a supercomputer [51]. Therefore, minimizing CPU energy consumption is critical for overall savings in the energy costs of a data center. In this work, we focus on intelligent selection of the cores on a chip with variation for running parallel HPC applications such that the energy consumption of the chip is minimized given execution time constraints. To provide the necessary background, we discuss the variation in future chips, the programming systems that can mitigate the impact of heterogeneity on application performance, and performance mod- 
eling of the chip in the wake of heterogeneity. We then propose a cubic integer programming based model for optimal selection of cores on the chip used for running the parallel application. Because of the cubic terms involved in the formulation, the problem is particularly hard to solve and therefore, we propose schemes to convert it to integer program formulation. Performance of the proposed approach is compared with two heuristics. Our results show that intelligent selection of cores using integer programming can lead to significant savings in energy costs.

The paper is divided into 7 sections. In Section 2, we perform a survey of literature on energy optimizations for HPC workloads. In Section 3, we discuss process variation that leads to heterogeneity across the cores on a chip, and performance modeling for such chips. The proposed integer programming approach for core selection is given in Section 4. The evaluation setup is given in Section 5, which is followed by results and their analysis in Section 6. Finally, we conclude the paper with conclusions and future work in Section 7.

\section{Related Work}

In the past, the emphasis has been on minimizing the completion times of the HPC applications. However, such solutions could have excessive energy consumption and hence high energy costs for the data centers. Consequently, minimizing energy consumption has become a major challenge for high performance computing data centers, especially with the increase in the size of the data centers. Hence, minimizing energy consumption has been a subject of intensive research over the past several years.

Dynamic power consumption of a chip is known to be a function of the frequency of the chip [20]. Applications do not yield proportionate improvement in performance with the increase in frequency of the chip, and therefore frequency is scaled down to reduce the power consumption of the chip while having tolerable impact on the completion time of the application. Modern processor architectures allow users to control the frequency of the chip through DVFS modules. There have been many studies on the use of DVFS for energy efficient computing for HPC workloads [24, 34, 40, 44]. Rizvandi et al [38] make some observations on optimal frequency selection in DVFS-based energy consumption minimization. Etinski et al [17] present a model that predicts the upper bound on performance loss due to frequency scaling. They study how sensitivity of the application to frequency scaling together with cluster characteristics determines the effectiveness of DVFS for energy consumption optimization. Wang et al [52] propose an energy aware scheduling heuristic that studies the slack time of non-critical tasks, and extends their execution time (by using DVFS) to save energy without affecting the overall execution time of the job. Vishnu et al [50] leverage DVFS to use the slack in one-sided communication primitives of PGAS for energy efficiency.

Lower core frequency also leads to lower core temperatures. DVFS has also been used for controlling the temperature of the chips, which reduces the temperature of the hot spots, that is, the nodes with highest temperature in the data center. Lower temperature of the hot spots means reduction in the cooling energy required to keep the temperatures of the hot spots at the room temperature. In this way, DVFS can been used to reduce the cooling energy costs of the data center. There has been a significant amount of work on various strategies for reducing the cooling energy of HPC and non-HPC data centers $[8,9,37,45,46,53,54]$.

Energy efficiency has been studied extensively in the context of large scale cloud computing as well $[48,55]$. The richness of the literature on energy optimization for data centers establishes the importance of this work.

Recent processor architectures, such as IBM Power6 [10], IBM Power7 [12], AMD Bulldozer [4], Intel ${ }^{\circledR}$ Sandybridge [39], provide the user with the ability to control the power consumption of CPU, DRAM, etc. The ability to constrain the power consumption of nodes provides the flexibility to add more nodes to the data center while remaining within the same power budget. This is also called overprovisioning. In our previous work ([41, 42]), we have shown significant improvement in performance of a data center by using overprovisioning under a strict power budget. We have also shown the benefit of using integer linear programming methods for improving the performance of applications on chips with low voltage operation under a strict power budget [47]. In contrast, the focus of this work is on minimizing the energy consumption of the chips, which is an even harder problem to solve because of the cubic and quadratic terms involved in the formulation of the problem.

Previous work (e.g. [30]) has proposed heterogeneous chip designs that have custom designed cores for a given set of target workloads. Different cores are designed to cater to different classes of applications. On the contrary, heterogeneity in the low voltage chips is inherent in the manufacturing process. Integer linear programming has been used in the past in the context of homogeneous multiprocessor chips. Kadayif et al [25] use integer linear programming for determining the optimal number of cores that will be used in executing each nest in the code of array-intensive applications under energy and performance constraints. Power Aware Resource Manager, PARM, proposed by Sarood et al [41] uses Integer Linear Program (ILP) to schedule and determine the optimal allocation of power and compute nodes to jobs submitted to a data center. Venugopalan et al [49] propose the use of ILP for optimal task scheduling on multiprocessors. To the best extent of our knowledge, energy efficiency in the context of chips with low voltage operation has not been addressed before. 


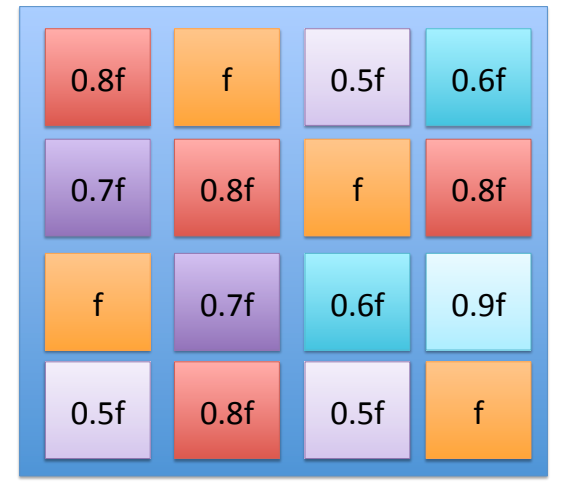

Figure 1: An example of core frequencies in a manycore chip with variation across cores.

\section{Preliminaries}

In this section, we review the causes of heterogeneity for future generation chips, and its impact on performance of parallel applications, such load imbalance. We then briefly study some of the programming systems that can overcome the impact on applications performance by performing load balancing of over-decomposed tasks. Finally we discuss some performance models that can predict an application's performance in such a heterogeneous environment. The performance models will be used in the next section (Section 4) for optimal selection of cores for energy-efficient computing. More details about these preliminaries can be found in previous work [47].

\subsection{Process Variation}

Operating at low voltage leads to increase in energy-efficiency of the chip. High energy efficiency of operation at low voltages has been established for $65,45,32,22 \mathrm{~nm}$ technologies [7, 22, 28, 29]. Kaul et al [27, 29] show that as the supply voltage of the transistor is reduced, the energy efficiency increases, and is maximum near the threshold voltage of the transistor. At threshold voltage, energy efficiency is $10 \times$ as compared to at the nominal supply voltage. However, as the supply voltage reaches near the threshold voltage, even a small change in the supply voltage leads to large spread in the frequency of operation. Therefore, different cores will be operating at different frequencies in a manycore chip. Leakage power also varies significantly across chips. More challenges associated with low voltage operation can be found in [27]. Nearest frequency of operation is assigned to these cores as shown in Figure 1.

\subsection{Programming Systems}

HPC applications are highly synchronized applications. For example, in many applications all the processors synchronize after every iteration (or every few iterations) to exchange neighbor boundaries. Hence, the speed of execution of a parallel HPC application is only as fast as the speed of the slowest processor. Of course, this is true only if the workload is distributed equally to the processors. When the processors have different speeds, the work load assigned to a core should be proportionate to its speed of operation. In order to do so, the total work has to be over-decomposed into many small tasks (more than the number of cores), such that it can be evenly distributed to the cores in proportion to their frequencies. It is not always possible to ensure load balance in such a situation. For example, if there are two processors with frequencies $f$ and $0.75 f$, and there are three equal sized tasks, then it is impossible to achieve perfect load balance. However, as the total number of tasks increases, the load imbalance decreases (provided an intelligent algorithm for load distribution is being used). Previous work [47] has shown that with an over-decomposition level of 16 (that is, the number of tasks to number of cores ratio is 16), the load imbalance can be contained to within $2-6 \%$ of the total execution time of the application. There are many parallel programming languages that over-decompose the total work into many small tasks. Some examples of such distributed parallel programming languages are Charm++ [6], AMPI [23], etc. For shared memory machines, Cilk [11], OpenMP [16], etc. are some examples of programming models in which the work is divided into chunks (for example, iterations in for loops in OpenMP) that can be dynamically assigned to processors during runtime.

For our proposed method, no changes are required either in the programming language or in the code (except possibly the addition/use of a variation aware load balancer).

\subsection{Performance Modeling}

In this section, we discuss the models to predict the performance (execution time or instructions per cycle) of a parallel HPC application on any configuration of a heterogeneous manycore chip. A configuration is a subset of the cores on the chip on which the parallel application will be executed. Other cores on the chip are turned-off so that they do not consume any static power. A good configuration of the chip for a given HPC application minimizes the total energy consumption during the execution period of the application. It is practically infeasible to evaluate all possible configurations of the chip for every application because the total number of configurations is combinatorially large. For example, when the number of cores on the chip is 36 , the total number of possible configurations is $2^{36}-1 \approx 6.87 e 10$. Therefore, performance models are required that can predict the performance of an application for any configuration. The model should require minimal profiling information of the application to be collected, so that the overhead of developing the performance models is negligible.

We now review the performance models for manycore heterogeneous chips from previous work [47]:

Model 1: All the cores can be individually profiled for the application, and the performance for a given configuration could be modeled as the sum of the performance of the 
individual cores in the configuration $(c)$.

$$
S=\sum_{i \in c} s_{i}
$$

where, $s_{i}$ is the performance (instructions per cycle) of core $i$ for the focal application when the application was run only on core $i$, and $S$ is the predicted performance (instructions per cycle) for configuration $c$ for the focal application. This model will predict performance accurately only for computationally intensive applications in which there is no memory contention. For memory-intensive applications, this performance model will fail to predict the performance for a configuration because it just adds the core performance which was obtained when they were running individually, and does not model the contention for the shared resources, e.g. memory, when multiple cores are running simultaneously.

Model 2: The application execution time is divided into two components: $T_{c p u}$ corresponding to CPU time and $T_{m e m}$ corresponding to memory time (as in $[14,15,21$, 43]). And the performance is modeled as

$$
T=\frac{T_{c p u}}{\sum_{i \in c} f_{i}}+T_{m e m}
$$

where, $f_{i}$ is the frequency of core $i$, and $T$ is the predicted execution time of the application. The weakness of this model is that it fails to incorporate the number of cores that are accessing the memory, and treats the memory time as constant irrespective of the cores that are accessing the memory.

Model 3: In this model, we construct as many model functions as there are number of cores on the chip. There is one model for all the configurations with the same number of cores. For instance, if there are 36 cores on a chip, 36 functions are developed. In this way, this model incorporates the number of active cores in performance prediction. Each of these functions is a linear function of the sum of frequencies of the cores in the configuration. The performance (instructions per cycle) function for all the configurations with $k$ cores is modeled as:

$$
S=a_{k}\left(\sum_{i \in c} f_{i}\right)+b_{k}
$$

where, $a_{k}, b_{k}$ are line constants for all configurations with $k$ cores, and $S$ is the instructions per cycle of the configuration. Only two performance data samples are required to get the value of the constants, $a_{k}$ and $b_{k}$, for this function. These samples correspond to instructions per cycle for any two configurations with $k$ cores. Since there are $n$ functions, $2 n$ samples are sufficient to develop the complete model for an application (although more samples can increase the accuracy of the model). The overhead of sampling the data to generate the model is negligible as compared to the execu-

\begin{tabular}{|c|c|}
\hline Symbol & Description \\
\hline$N$ & total number of cores on the chip \\
\hline$c$ & a configuration \\
\hline$k$ & number of cores in a configuration \\
\hline$n_{k}$ & $\begin{array}{l}\text { binary variable indicating whether the selected } \\
\text { configuration has } k \text { cores }\end{array}$ \\
\hline$x_{i}$ & $\begin{array}{l}\text { a binary variable indicating whether core } i \\
\text { is selected or not in a configuration }\end{array}$ \\
\hline$f_{i}$ & frequency of core $i$ \\
\hline & static power consumption of core $i$ \\
\hline$a_{k}^{t}, b_{k}^{t}$ & $\begin{array}{l}\text { line constants for performance model } \\
\text { of configurations with } k \text { cores }\end{array}$ \\
\hline$a_{k}^{p}, b_{k}^{p}$ & $\begin{array}{l}\text { line constants for dynamic power model } \\
\text { of configurations with } k \text { cores }\end{array}$ \\
\hline$t_{\min }$ & $\begin{array}{l}\text { minimum execution time of the application } \\
\text { across all the configurations on the chip }\end{array}$ \\
\hline$t p$ & $\begin{array}{l}\text { penalty in execution time, maximum allowed } \\
\text { execution time is }\left(1+\frac{t p}{100}\right) \times t_{\min }\end{array}$ \\
\hline
\end{tabular}
tion time of HPC applications, which can be from hours to
Table 1: Constrained Optimization Program Terminology

days. In previous work [47], it is shown that the prediction accuracy of Model 3 is very high. The average prediction error in performance is less than $1.6 \%$, and $0.7 \%$ for a computationally intensive and a memory intensive application, respectively. Simulated performance was obtained using the Sniper simulator, discussed in detail in Section 5. Similar to performance, the dynamic power consumption of a configuration could be modeled accurately using Model 3, that is,

$$
P=A_{k}\left(\sum_{i \in c} f_{i}\right)+B_{k}
$$

where $P$ is the dynamic power of configuration $c, A_{k}$ and $B_{k}$ are line constants. It has been shown in previous work [47] that the maximum prediction error of Model 3 for dynamic power is less than $2 \%$.

\section{Energy Optimization Approach}

In this section, we describe our approach for optimizing the energy consumption during application execution. The total energy is computed as the power consumption integrated over the duration of execution of the application, that is, power consumption multiplied by the execution time of the application. We use Model 3, described in the previous section, to model the execution time and dynamic power consumption of any configuration. According to Model 3, the linear function for performance and dynamic power consumption of a configuration depends on the number of cores in the configuration. Therefore, the energy consumption can be defined as

$\sum_{k=1}^{N}\left(n_{k} *\left(a_{k}^{p} \sum_{i} x_{i} f_{i}+b_{k}^{p}+\sum_{i} s_{i} x_{i}\right) *\left(a_{k}^{t} \sum_{i} x_{i} f_{i}+b_{k}^{t}\right)\right)$ 
where, $n_{k}$ is a binary variable indicating whether the selected configuration has $k$ cores $\left(n_{k}\right.$ can be 1 only for one value of $k), x_{i}$ is a binary variable indicating whether $i^{\text {th }}$ core is selected, $a_{k}^{p} \sum_{i} x_{i} f_{i}+b_{k}^{p}$ is the dynamic power consumption of the configuration, $s_{i}$ is the static power consumption of core $i, \sum_{i} s_{i} x_{i}$ is the total static power consumption, and $a_{k}^{t} \sum_{i} x_{i} f_{i}+b_{k}^{t}$ is the execution time of the application. Energy minimization problem can then be formulated as a constrained optimization problem. The formulation is given below in Equations (4)-(8). Terminology used in this section is defined in Table 1.

Objective Function $\overline{\text { Algorithm } 1 \text { Algorithm for obtaining the globally optimal }}$ configuration by solving $n$ quadratic programs

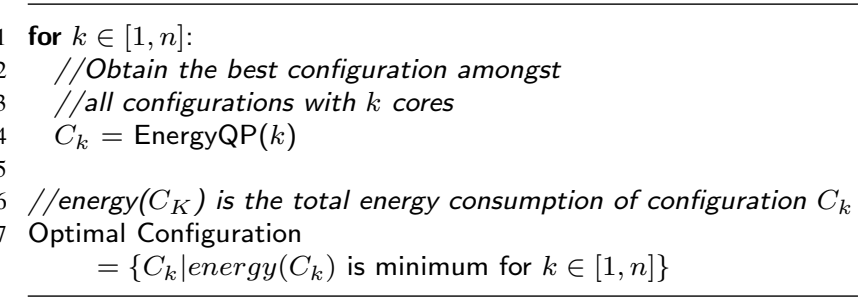

\section{Total Number of Cores Equals K}

$$
\sum_{i=0}^{n-1} x_{i}=K
$$

Variables Range
Select One Value of $k$

$$
\sum_{k=1}^{n} n_{k}=1
$$

Total Number of Cores Equals $k$

$$
\sum_{i=0}^{n-1} x_{i}=\sum_{k=1}^{n} n_{k} k
$$

Variables Range

$$
\begin{array}{ll}
\forall i \in[0, n), & x_{i} \in\{0,1\} \\
\forall k \in(0, n], & n_{k} \in\{0,1\}
\end{array}
$$

Constraints in the above formulation are linear constraints that ensure that a valid configuration is selected. However, the objective function has a cubic expression. This constrained optimization problem can be readily solved by solving $n$ quadratic integer programs. Each of these quadratic integer programs chooses the best configuration amongst all the configurations with the same number of cores. The best performing configuration is then chosen from amongst the optimal configurations returned by the $n$ quadratic integer program optimizations. In this way, the global optimal configuration can be found by optimizing $n$ quadratic programs (Algorithm 1). The quadratic program that selects the best configuration from amongst all the configurations with $k$ cores is given below in Equations (9)-(11).

Objective Function

$\min \left(a_{K}^{p} \sum_{i=0}^{n-1} x_{i} f_{i}+b_{K}^{p}+\sum_{i=0}^{n-1} s_{i} x_{i}\right) *\left(a_{K}^{t} \sum_{i=0}^{n-1} x_{i} f_{i}+b_{K}^{t}\right)$

$$
\forall i \in[0, n), \quad x_{i} \in\{0,1\}
$$

Quadratic programs must have positive semi-definite matrices to be solved using convex optimization. The resulting quadratic programs above are not positive semi-definite and hence can be computationally very hard to solve using nonlinear optimization methodologies.

In order to reduce the quadratic objective function to a linear expression, we use the scheme proposed by Glover and Woosley [19]. In this scheme, the cross-product terms in the objective function are replaced by adding new continuous variables. The value of these new variables are determined by adding new constraints. For example, a quadratic product term $x_{1} x_{2}$, where $x_{1}, x_{2}$ are binary variables, can be replaced by a new variable $y_{12}$ such that $y_{12} \leq x_{1}, y_{12} \leq$ $x_{2}$, and $y_{12} \geq x_{1}+x_{2}-1$. We multiply the terms in the objective function (Equation 9) and replace the product terms of the form $x_{i} x_{j}$ with new continuous variables $y_{i j}$. The resulting ILP is given below in Equations (12)-(15).

Objective Function

$$
\begin{array}{r}
\min \sum_{i=0}^{n-1} \sum_{j=0}^{n-1}\left(a_{K}^{p} f_{i}+s_{i}\right)\left(a_{K}^{t} f_{j}\right) y_{i j}+b_{K}^{t} \sum_{i=0}^{n-1}\left(a_{K}^{p} f_{i}+s_{i}\right) x_{i} \\
+b_{K}^{p} a_{K}^{t} \sum_{j=0}^{n-1} f_{j} x_{j}+b_{K}^{p} b_{K}^{t}
\end{array}
$$

Total Number of Cores Equals K

$$
\sum_{i=0}^{n-1} x_{i}=K
$$

New variable constraints

$$
\begin{array}{cc}
y_{i j} \leq x_{i}, & \forall i, j \in[0, n), j \leq i \\
y_{i j} \leq x_{j}, & \forall i, j \in[0, n), j \leq i \\
y_{i j} \geq x_{i}+x_{j}-1, & \forall i, j \in[0, n), j \leq i
\end{array}
$$


Variables Range

$$
\forall i \in[0, n), \quad x_{i} \in\{0,1\}
$$

This approach increases the number of variables $v$ by a factor of $\frac{v(v-1)}{2}$, and the number of constraints by a factor of $\frac{3 v(v-1)}{2}$. Since the value of $v$ is small for the focal problem, the size of the linear program remains tractable. It is possible that the configuration with minimum energy consumption has a very large execution time as compared to best execution time. In order to constrain the increase in execution time, the following time constraint is added to the linear programs, where $t p$ is the allowed increase in execution time.

$$
a_{K}^{t}\left(\sum_{i=0}^{n-1} x_{i} f_{i}\right)+b_{K}^{t} \leq\left(1+\frac{t p}{100}\right) * t_{\text {min }}
$$

This proposed ILP methodology is evaluated in Section 6.

\section{The Setup}

We use Sniper Multi-core Simulator [13] for simulating chips with heterogeneity. We use the default core model of Sniper. The default core model is similar to Intel ${ }^{\circledR}$ Gainestown model and has been validated [13]. We simulate chips with 36 cores. Each chip has x86 cores with 4-wide out-of-order issues. Each core has a private 4-way L1 Instruction cache of size $32 \mathrm{~KB}$, and a private 8-way L2 cache of size $256 \mathrm{~KB}$. There is a shared 4-way L1 Instruction cache of size $32 \mathrm{~KB}$. The memory latency is $75 \mathrm{~ns}$, when there is no memory contention. We use 11-nm technology, with average frequency of $2.6 \mathrm{GHz}$, and $0.765 V_{d d}$. McPAT (Multicore Power, Area, and Timing) [33] framework was integrated with Sniper for dynamic power modeling of manycore applications. Our experimental results in this paper are for chips with 36 cores. For modeling process variation at micro-architectural level and for static power modeling of cores, we use VariusNTV [26]. 25 chips were generated with different core frequencies and static power consumption. The frequencies and the corresponding static power consumption of various cores for one of the chips is shown in Figure 2.

\subsection{Applications}

Two HPC applications are used for benchmarking the performance:

- miniMD: It is a simple, parallel molecular dynamics code that is a micro-application in the Mantevo project at Sandia National Laboratories ${ }^{1}$. miniMD is written in MPI and performs parallel molecular dynamics simulation of a Lennard-Jones system. miniMD is a computationally intensive application.

\footnotetext{
${ }^{1}$ http://software.sandia.gov/mantevo/
}

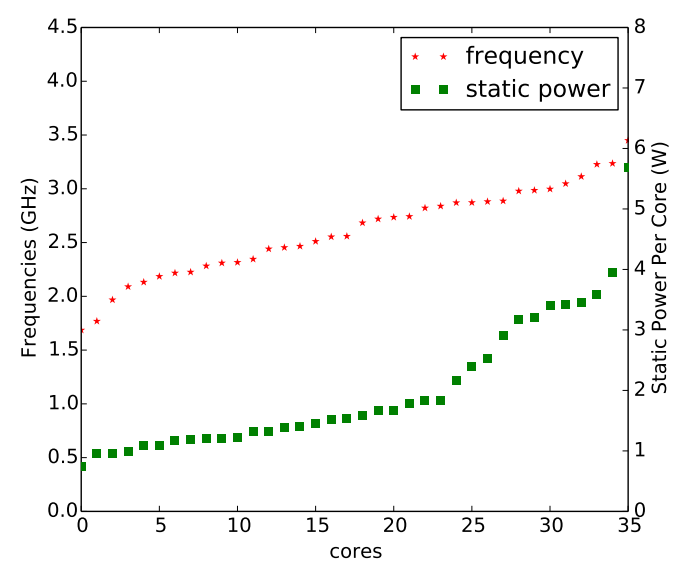

Figure 2: Frequencies and static powers of various cores on a manycore chip with variation.

- Jacobi: Jacobi is a 3D stencil computation code. It is a memory intensive application. We use a Charm++ implementation of Jacobi.

Most other HPC applications fall in between miniMD and Jacobi in terms of their computation and memory sensitivity. For developing the performance and dynamic power consumption models of these applications, we obtained $2 n$ samples from each application, where $n$ is the number of cores on the chip. For each value of $k \in[1, n], 2$ samples are required to build the linear model for all configurations with $k$ cores. We chose the configurations with $k$ minimum, and $k$ maximum frequency chips, and obtained their simulated performance on the Sniper simulator. The simulated performance was used to compute the line constants for the performance and power consumption models.

We use the reference energy consumption (refenergy) as the energy consumption corresponding to the configuration with the best possible execution time $\left(t_{\text {min }}\right)$, computed using Algorithm 2. Our results are compared against refenergy.

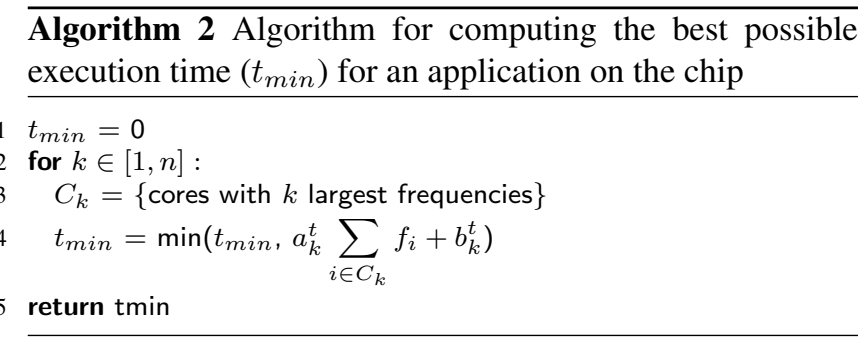

\subsection{Heuristics}

The proposed integer linear programming approach for energy minimization is compared against two heuristics, called the MIN, and MAX heuristics as described below:

- Min heuristic: The cores are sorted in the increasing order of their frequencies, such that, $f_{0}<f_{1}<f_{2} \ldots<$ 
$f_{n-1}$. The heuristic selects the value of $k$ such that the configuration with $k$ consecutive cores, starting from core $_{0}$ has the minimum energy consumption and the execution time is within the desirable threshold $(t p)$. The MIN heuristic algorithm is given in Algorithm 3.

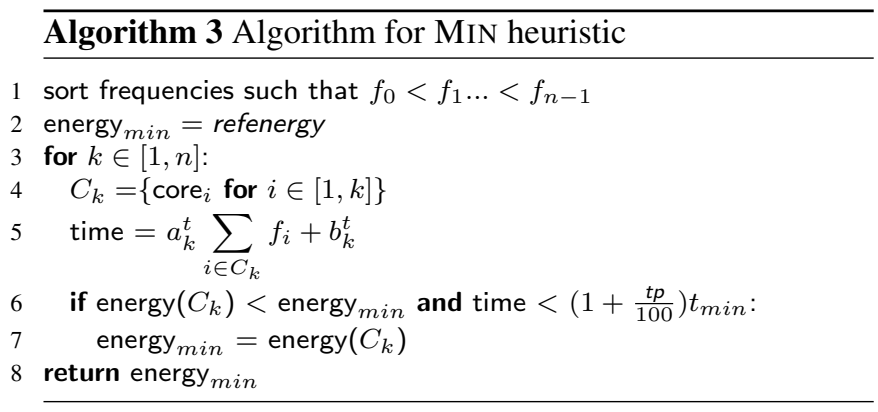

- MAX heuristic: The cores are sorted in the decreasing order of their frequencies, such that, $f_{0}>f_{1} \ldots . .>f_{n-1}$. The heuristic selects the value of $k$ such that the configuration with $k$ consecutive cores, starting from core ${ }_{0}$ has the minimum energy consumption and the execution time is within the desirable threshold $(t p)$. The MAX heuristic algorithm is given in Algorithm 4.

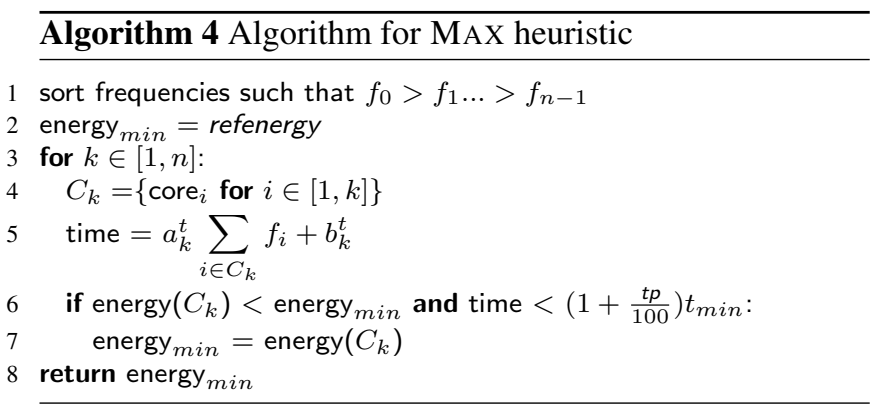

\subsection{ILP Solver}

There are several solvers available for integer linear program optimization, such as, Gurobi [1], CPLEX [2], GLPK [36], CBC [18], SCIP [5], Xpress [32]. We use the commercial state-of-the-art solver, Gurobi, for solving the Integer Linear Programs (ILPs). ILPs are NP-hard problems and are solved by using variants and extensions of Branch-andBound $(\mathrm{BnB})$ method. In BnB method, the corresponding linear program, obtained by relaxing the integrality constraints on integer variables, is first solved by using the simplex or the interior point method. This gives a fractional solution. Branching is done on the fractional values, which gives more linear programs. Linear program optimizations are done and the branching is continued until an integer solution is found. The integer solution with the best cost acts as an incumbent solution and is used to prune other vertices of the $\mathrm{BnB}$ tree that can provably be shown to not have better cost than the current incumbent. Commercial state-of-the art solvers like Gurobi have highly optimized implementations for solving ILPs. They fully exploit the latest mathematical and engineering improvements in the underlying methodologies to provide very fast solutions to linear/mixed-integer programs. Solvers like Gurobi are used for variety of cost and quality optimization purposes in various fields of optimization.

\section{Results}

In this section, we discuss the energy savings obtained using the ILP methodology and the optimization times of the ILPs.

\subsection{Energy-efficiency}

Figure 3 shows the savings in energy consumption by using configurations selected by the MIN heuristic, MAX heuristic, and the proposed integer linear programming method when compared to the configuration with best execution time. The results are summary of benefits across 25 different chips. We consider 3 cases, corresponding to Figure $3 a, 3 b, 3 c$, respectively.

1. In this case, configuration with minimum energy consumption is sought using the three methods without any limit on the increase in execution time (i.e. $t p \rightarrow \infty$ ).

- miniMD The ILP method gives an average of $26 \%$ in energy savings, while the savings were $9.8 \%$ and 4.7\% from MIN, and MAX heuristics, respectively.

- Jacobi We obtain an average of $10.77 \%$ savings in energy consumption using the ILP method. On the other hand, MIN and MAX heuristics give energy savings of only $5.45 \%$ and $1.52 \%$, respectively.

Although ILP gives significant savings in energy, it leads to significant increase in execution time. For instance, for miniMD there was an average of $74 \%, 10 \%, 156 \%$ increase in execution time with MIN, MAX heuristic, ILP, respectively. Therefore, we consider the following two cases in which there is a limit to the increase in execution time.

2. When the execution time is less than $1.15 \times t_{\min }$, that is, $t p=15 \%$

- miniMD We obtain an average of 4.8\%, 4.4\%, 18.4\% savings in energy with MIN, MAX heuristic, ILP, respectively.

- Jacobi An average of 2.9\%, 1.5\%, 8.6\% savings in energy with MIN, MAX heuristic, ILP, respectively is obtained.

3. When the execution time is less than $1.05 \times t_{\min }$, that is, $t p=5 \%$

- miniMD We obtain an average of $0.2 \%, 3.5 \%, 13.4 \%$ savings in energy with MIN, MAX heuristic, ILP, respectively. 


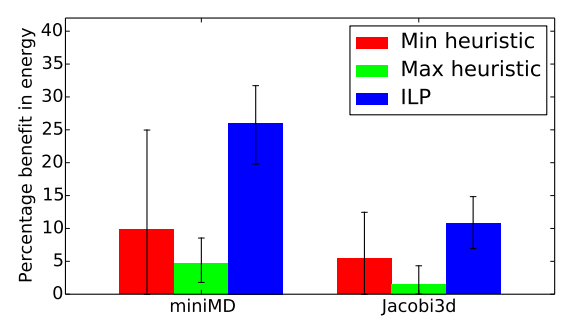

(a) With no time constraint

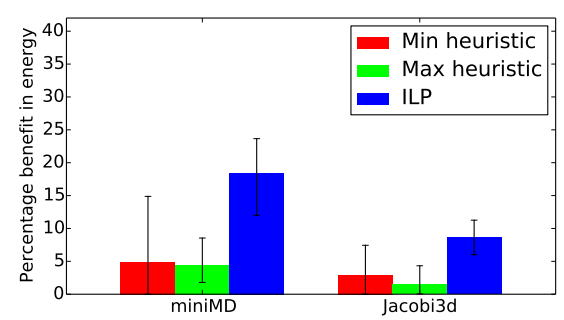

(b) Maximum $15 \%$ time penalty

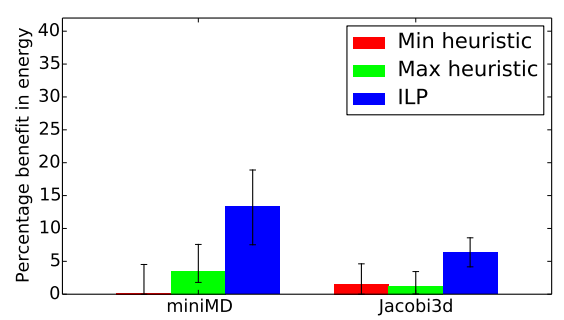

(c) Maximum 5\% time penalty

Figure 3: Percentage savings in energy with MIN, MAX heuristics, and the ILP method for the two applications, miniMD and Jacobi3d, with respect to the configuration with best execution time. The bars correspond to the average benefits, while the vertical lines correspond to the minimum and maximum benefits obtained from the corresponding heuristic across the 25 chips. In (a), configuration that minimizes energy consumption is sought irrespective of penalty in execution time of the application. In (b) and (c), the best configuration that minimizes energy while the execution time penalty is less than $15 \%$ and $5 \%$, respectively, is sought using the MIN, MAX heuristic, and ILP method.

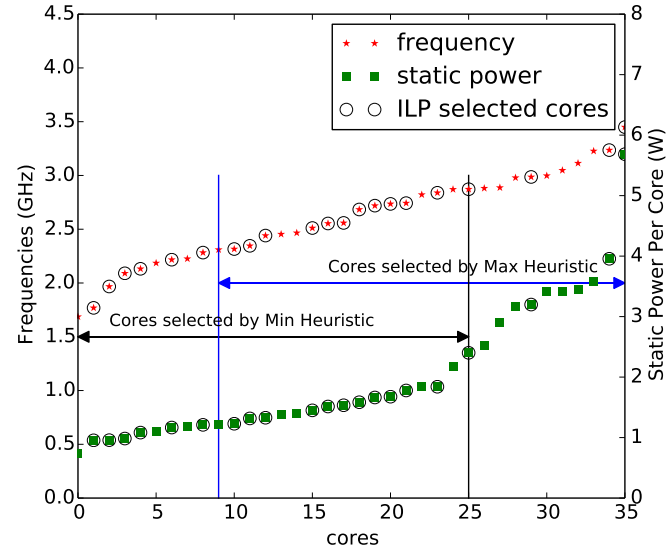

Figure 4: An example of a configuration selected by the ILP optimization method for Jacobi application. Circle markers correspond to the cores selected by ILP. A total of 21 cores were selected by the ILP method. MIN, MAX heuristic selected 26, 27 cores, respectively.

- Jacobi An average of $1.6 \%, 1.2 \%, 6.4 \%$ savings in energy with MIN, MAX heuristic, ILP, respectively is achieved.

Since miniMD is a computationally intensive application, the number of cores in the optimal configuration selected for miniMD are more than the number of cores in the optimal configuration for Jacobi. In Jacobi, large number of cores lead to increase in the memory contention and hence are suboptimal. Figure 4 shows an example solution obtained from ILP optimization, MIN heuristic, and MAX heuristic.

\subsection{Solution Time}

The proposed methodology requires optimizing $n-2$ proper ILPs, where $n$ is the total number of cores on the chip. Each ILP has 702 variables, and 2000 constraints. The ILP optimizations are independent of each other and can therefore be very easily parallelized by launching them in parallel on multiple cores of a compute node and/or on multiple compute nodes. For the experiments, we use a Dell $2.67 \mathrm{GHz}$ Dual Westmere Xeon E5640 processor with a total of 8 cores and 16 SMT threads. The ILP optimizations required for a given chip and a application were launched in parallel on the machine. We now consider the solution time for each of the three cases presented in Section 6.1.

1. When there is no execution time penalty constraint, it took an average of 400 seconds and 1090 seconds for obtaining the optimal result for miniMD and Jacobi, respectively. An average of $4.08 e 7$ and $3.27 e 8$ simplex iterations (summed across all the $\mathrm{BnB}$ vertices explored) were performed by the ILP solver for miniMD and Jacobi, respectively.

2. When the maximum execution time penalty of $15 \%$ is enforced, the configuration search space for ILP optimization is reduced significantly. It took an average of $14.8 \mathrm{~s}$, 37 s to find the optimal solution for miniMD, Jacobi, respectively.

3. With the maximum execution time penalty of $5 \%$, the search space is further reduced, and it took only $8 \mathrm{~s}, 10.2 \mathrm{~s}$ to find the optimal solution for miniMD, Jacobi, respectively.

We compare these results with exhaustive evaluation of the performance and power models for all the configurations on the same machine. The configurations can be evaluated in parallel. The total number of configurations to be evaluated 
for a 36 core chip is $2^{36}-1$, which is equal to 68719476736 configurations. Exhaustive evaluation of all these configurations in parallel on 8 cores takes 74 hours. These results show the benefit of using ILP solvers. The time to solution for the ILP method can be significantly reduced further by using a compute node with more cores, or using multiple nodes for parallel optimization. Since HPC simulations run for several hours, the overhead of finding the optimal configuration is negligible as compared to the execution time of the jobs, which can be from hours to days.

\section{Conclusion and Future Work}

We studied chips with variation - the heterogeneity across their cores, their performance modeling, and runtime system. We proposed an integer linear programming based methodology to obtain a configuration of the chip that optimizes the energy consumption for a given application that originally has a hard-to-solve quadratic integer program formulation. Our results show that as much as $26 \%$ savings in energy can be obtained for some applications by using the proposed methodology. On the other hand, exhaustive evaluation of all the configurations is intractable for practical purposes. Using the proposed method, the overhead of performance modeling and optimal configuration selection is negligible. Whenever a job is scheduled for execution on selected chips, the ILP optimizer can be executed on the chip itself to determine the optimal configuration for the job, prior to actual execution of the job on that chip. In this way, no extra compute resources are required for optimal configuration selection.

There is significant future work that ensues from here. We plan to evaluate the proposed approach for chips with very large number of cores. We also wish to explore optimization techniques which can further reduce the solution time for finding the optimal configurations. We plan to evaluate the proposed methodology for other applications that are both computationally and memory intensive such as Adaptive Mesh Refinement [31], Lulesh [35], etc.

\section{Acknowledgments}

The authors, Akhil Langer, Udatta Palekar, and L.V. Kale's work is partially supported by MITRE Research Agreement Number 81990 with UIUC.

\section{References}

[1] Gurobi Optimization Inc. Software, 2014. http://www. gurobi.com/.

[2] IBM CPLEX Optimization Studio. Software, 2012. http://www-01.ibm.com/software/integration/ optimization/cplex-optimization-studio/.

[3] The Department of Energy Report on Top Ten Exascale Research Challenges. http://science.energy. gov/ /media/ascr/ascac/pdf/meetings/20140210/ Top10reportFEB14.pdf.
[4] Advanced Micro Devices. BIOS and Kernel Developer's guide (BKDG) for AMD Family $15 \mathrm{~h}$ Models 00h-0fh Processors. January 2012.

[5] T. Achterberg. SCIP: Solving Constraint Integer Programs. Mathematical Programming Computation, 1(1):1-41, 2009.

[6] B. Acun, A. Gupta, N. Jain, A. Langer, H. Menon, E. Mikida, X. Ni, M. Robson, Y. Sun, E. Totoni, L. Wesolowski, and L. Kale. Parallel Programming with Migratable Objects: Charm++ in Practice. In Proceedings of the International Conference on High Performance Computing, Networking, Storage and Analysis, SC '14, New York, NY, USA, 2014. ACM.

[7] A. Agarwal, S. K. Mathew, S. K. Hsu, M. A. Anders, H. Kaul, F. Sheikh, R. Ramanarayanan, S. Srinivasan, R. Krishnamurthy, and S. Borkar. A 320mV-to-1.2 V On-die Finegrained Reconfigurable Fabric For DSP/media Accelerators in 32nm CMOS. In Solid-State Circuits Conference Digest of Technical Papers (ISSCC), 2010 IEEE International, pages 328-329. IEEE, 2010.

[8] A. Banerjee, T. Mukherjee, G. Varsamopoulos, and S. Gupta. Cooling-aware and thermal-aware workload placement for green HPC data centers. In 2010 International Green Computing Conference, pages 245-256, August 2010.

[9] C. Bash and G. Forman. Cool job allocation: measuring the power savings of placing jobs at cooling-efficient locations in the data center. In Proceedings of the USENIX Annual Technical Conference, pages 29:1-29:6, Berkeley, CA, USA, 2007. USENIX Association.

[10] B. Behle, N. Bofferding, M. Broyles, C. Eide, M. Floyd, C. Francois, A. Geissler, M. Hollinger, H.-Y. McCreary, C. Rath, et al. IBM Energyscale for POWER6 Processorbased Systems. IBM White Paper, 2009.

[11] R. D. Blumofe, C. F. Joerg, B. C. Kuszmaul, C. E. Leiserson, K. H. Randall, and Y. Zhou. Cilk: An efficient multithreaded runtime system. Journal of Parallel and Distributed Computing, 37(1):55-69, 1996.

[12] M. Broyles, C. Francois, A. Geissler, M. Hollinger, T. Rosedahl, G. Silva, J. Van Heuklon, and B. Veale. IBM Energyscale for POWER7 Processor-based Systems. white paper, IBM, 2010.

[13] T. E. Carlson, W. Heirman, and L. Eeckhout. Sniper: exploring the level of abstraction for scalable and accurate parallel multi-core simulation. In Proceedings of 2011 International Conference for High Performance Computing, Networking, Storage and Analysis, page 52. ACM, 2011.

[14] K. Choi, R. Soma, and M. Pedram. Dynamic Voltage and Frequency Scaling based on Workload Decomposition. In Proceedings of the 2004 international symposium on Low power electronics and design, pages 174-179. ACM, 2004.

[15] K. Choi, R. Soma, and M. Pedram. Fine-grained Dynamic Voltage and Frequency Scaling for Precise Energy and Performance Tradeoff based on the Ratio of Off-chip Access to On-chip Computation Times. Computer-Aided Design of Integrated Circuits and Systems, IEEE Transactions on, 24(1):1828, 2005. 
[16] L. Dagum and R. Menon. OpenMP: an industry standard API for shared-memory programming. Computational Science \& Engineering, IEEE, 5(1):46-55, 1998.

[17] M. Etinski, J. Corbalan, J. Labarta, and M. Valero. Understanding the Future of Energy-performance Trade-off via DVFS in HPC Environments. Journal of Parallel and Distributed Computing, 72(4):579-590, 2012.

[18] J. Forrest. CBC (Coin-or Branch and Cut) Open-source Mixed Integer Programming Solver, 2012. URL https://projects.coinor.org/Cbc.

[19] F. Glover and E. Woolsey. Converting the 0-1 Polynomial Programming Problem to a 0-1 Linear Program. Operations Research, 22(1):pp. 180-182, 1974.

[20] R. Graybill and R. Melhem. Power aware computing. Kluwer Academic Publishers, 2002.

[21] C.-H. Hsu and W.-c. Feng. Effective dynamic voltage scaling through cpu-boundedness detection. In Power-Aware Computer Systems, pages 135-149. Springer, 2005.

[22] S. Hsu, A. Agarwal, M. Anders, H. Kaul, S. Mathew, F. Sheikh, R. Krishnamurthy, and S. Borkar. A $2.8 \mathrm{GHz} 128-$ entry $\times 152 \mathrm{~b} 3$-read/2-write Multi-precision Floating-point Register File and Shuffler in 32nm CMOS. In VLSI Circuits (VLSIC), 2012 Symposium on, pages 118-119. IEEE, 2012.

[23] C. Huang, G. Zheng, S. Kumar, and L. V. Kalé. Performance Evaluation of Adaptive MPI. In Proceedings of ACM SIGPLAN Symposium on Principles and Practice of Parallel Programming 2006, March 2006.

[24] S. Huang and W. Feng. Energy-efficient cluster computing via accurate workload characterization. In Proceedings of the 2009 9th IEEE/ACM International Symposium on Cluster Computing and the Grid, CCGRID '09, pages 68-75, Washington, DC, USA, 2009. IEEE Computer Society.

[25] I. Kadayif, M. Kandemir, and U. Sezer. An integer linear programming based approach for parallelizing applications in on-chip multiprocessors. In Proceedings of the 39th annual Design Automation Conference, pages 703-706. ACM, 2002.

[26] U. R. Karpuzcu, K. B. Kolluru, N. S. Kim, and J. Torrellas. VARIUS-NTV: A microarchitectural model to capture the increased sensitivity of manycores to process variations at near-threshold voltages. In Dependable Systems and Networks (DSN), 2012 42nd Annual IEEE/IFIP International Conference on, pages 1-11. IEEE, 2012.

[27] H. Kaul, M. Anders, S. Hsu, A. Agarwal, R. Krishnamurthy, and S. Borkar. Near-threshold Voltage (NTV) Design: Opportunities and Challenges. In Proceedings of the 49th Annual Design Automation Conference, pages 1153-1158. ACM, 2012.

[28] H. Kaul, M. Anders, S. Mathew, S. Hsu, A. Agarwal, R. Krishnamurthy, and S. Borkar. A 300mV 494GOPS/W Reconfigurable Dual-supply 4-Way SIMD Vector Processing Accelerator in 45nm CMOS. In IEEE International Solid-State Circuits Conference, ISSCC 2009, Digest of Technical Papers, San Francisco, CA, USA, 8-12 February, 2009, pages 260261, 2009.

[29] H. Kaul, M. A. Anders, S. K. Mathew, S. K. Hsu, A. Agarwal, R. K. Krishnamurthy, and S. Borkar. A $320 \mathrm{mv} 56 \mu \mathrm{w} 411$ gops/watt Ultra-low Voltage Motion Estimation Accelerator in $65 \mathrm{~nm}$ CMOS. Solid-State Circuits, IEEE Journal of, 44(1):107-114, 2009.

[30] R. Kumar, D. M. Tullsen, and N. P. Jouppi. Core architecture optimization for heterogeneous chip multiprocessors. In Proceedings of the 15th international conference on Parallel architectures and compilation techniques, pages 23-32. ACM, 2006.

[31] A. Langer, J. Lifflander, P. Miller, K.-C. Pan, , L. V. Kale, and P. Ricker. Scalable Algorithms for Distributed-Memory Adaptive Mesh Refinement. In Proceedings of the 24th International Symposium on Computer Architecture and High Performance Computing (SBAC-PAD 2012), New York, USA, October 2012.

[32] R. Laundy, M. Perregaard, G. Tavares, H. Tipi, and A. Vazacopoulos. Solving Hard Mixed-integer Programming Problems with Xpress-MP: A MIPLIB 2003 Case Study. INFORMS Journal on Computing, 21(2):304-313, 2009.

[33] S. Li, J. H. Ahn, R. D. Strong, J. B. Brockman, D. M. Tullsen, and N. P. Jouppi. Mcpat: an integrated power, area, and timing modeling framework for multicore and manycore architectures. In Microarchitecture, 2009. MICRO-42. 42nd Annual IEEE/ACM International Symposium on, pages 469-480. IEEE, 2009.

[34] M. Y. Lim, V. W. Freeh, and D. K. Lowenthal. Adaptive, transparent CPU scaling algorithms leveraging inter-node MPI communication regions. Parallel Computing, 37(10-11):667683, 2011.

[35] Lulesh. http://computation.llnl.gov/casc/ ShockHydro/.

[36] A. Makhorin. The GNU Linear Programming Kit (GLPK). GNU Software Foundation, 2000. http://www.gnu.org/ software/glpk/glpk.html.

[37] A. Merkel and F. Bellosa. Balancing power consumption in multiprocessor systems. In Proceedings of the 1st ACM SIGOPS/EuroSys European Conference on Computer Systems, EuroSys '06, pages 403-414, New York, NY, USA, 2006. ACM.

[38] N. B. Rizvandi, J. Taheri, and A. Y. Zomaya. Some Observations on Optimal Frequency Selection in DVFS-based Energy Consumption Minimization. Journal of Parallel and Distributed Computing, 71(8):1154-1164, 2011.

[39] B. Rountree, D. H. Ahn, B. R. de Supinski, D. K. Lowenthal, and M. Schulz. Beyond DVFS: A First Look at Performance Under a Hardware-enforced Power Bound. In IEEE 26th International Parallel and Distributed Processing Symposium Workshops \& PhD Forum (IPDPSW), 2012.

[40] B. Rountree, D. K. Lowenthal, S. Funk, V. W. Freeh, B. R. de Supinski, and M. Schulz. Bounding Energy Consumption in Large-scale MPI Programs. In Proceedings of the ACM/IEEE conference on Supercomputing, pages 49:1-49:9, 2007.

[41] O. Sarood, A. Langer, A. Gupta, and L. V. Kale. Maximizing throughput of overprovisioned hpc data centers under a strict power budget. In Proceedings of the International Conference 
on High Performance Computing, Networking, Storage and Analysis, SC '14, New York, NY, USA, 2014. ACM.

[42] O. Sarood, A. Langer, L. V. Kale, B. Rountree, and B. de Supinski. Optimizing Power Allocation to CPU and Memory Subsystems in Overprovisioned HPC Systems. In Proceedings of IEEE Cluster 2013, Indianapolis, IN, USA, September 2013.

[43] K. Seth, A. Anantaraman, F. Mueller, and E. Rotenberg. Fast: Frequency-aware Static Timing Analysis. ACM Transactions on Embedded Computing Systems (TECS), 5(1):200224, 2006.

[44] R. Springer, D. K. Lowenthal, B. Rountree, and V. W. Freeh. Minimizing execution time in MPI programs on an energyconstrained, power-scalable cluster. In Proceedings of the eleventh ACM SIGPLAN symposium on Principles and practice of parallel programming, PPoPP '06, pages 230-238, New York, NY, USA, 2006. ACM.

[45] Q. Tang, S. Gupta, D. Stanzione, and P. Cayton. Thermalaware task scheduling to minimize energy usage of blade server based datacenters. In 2nd IEEE International Symposium on Dependable, Autonomic and Secure Computing, 2006.

[46] Q. Tang, S. Gupta, and G. Varsamopoulos. Energyefficient thermal-aware task scheduling for homogeneous high-performance computing data centers: A cyber-physical approach. IEEE Transactions on Parallel and Distributed Systems, (11):1458-1472, November 2008.

[47] E. Totoni. Power and Energy Management of Modern Architectures in Adaptive HPC Runtime Systems. PhD thesis, Dept. of Computer Science, University of Illinois, 2014.

[48] G. L. Valentini, W. Lassonde, S. U. Khan, N. Min-Allah, S. A. Madani, J. Li, L. Zhang, L. Wang, N. Ghani, J. Kolodziej, et al. An overview of energy efficiency techniques in cluster computing systems. Cluster Computing, 16(1):3-15, 2013.

[49] S. Venugopalan and O. Sinnen. Optimal linear programming solutions for multiprocessor scheduling with communication delays. In Y. Xiang, I. Stojmenovic, B. Apduhan, G. Wang, K. Nakano, and A. Zomaya, editors, Algorithms and Architectures for Parallel Processing, volume 7439 of Lecture Notes in Computer Science, pages 129-138. Springer Berlin Heidelberg, 2012.

[50] A. Vishnu, S. Song, A. Marquez, K. Barker, D. Kerbyson, K. Cameron, and P. Balaji. Designing energy efficient communication runtime systems: a view from pgas models. The Journal of Supercomputing, 63(3):691-709, 2013.

[51] S. Wallace, V. Vishwanath, S. Coghlan, Z. Lan, and M. E. Papka. Measuring Power Consumption on IBM Blue Gene/Q. In Parallel and Distributed Processing Symposium Workshops \& PhD Forum (IPDPSW), 2013 IEEE 27th International, pages 853-859. IEEE, 2013.

[52] L. Wang, S. U. Khan, D. Chen, J. Kołodziej, R. Ranjan, C.-z. $\mathrm{Xu}$, and A. Zomaya. Energy-aware Parallel Task Scheduling in a Cluster. Future Generation Computer Systems, 29(7):1661-1670, 2013.

[53] L. Wang, G. von Laszewski, J. Dayal, and T. Furlani. Thermal aware workload scheduling with backfilling for green data centers. In Proceedings of the 2009 IEEE 28th International Performance Computing and Communications Conference (IPCCC), December 2009.

[54] L. Wang, G. von Laszewski, J. Dayal, X. He, A. Younge, and T. Furlani. Towards thermal aware workload scheduling in a data center. In International Symposium on Pervasive Systems, Algorithms, and Networks (ISPAN), December 2009.

[55] G. Wen, J. Hong, C. Xu, P. Balaji, S. Feng, and P. Jiang. Energy-aware Hierarchical Scheduling of Applications in Large Scale Data Centers. In Cloud and Service Computing (CSC), 2011 International Conference on, pages 158-165. IEEE, 2011. 